\title{
New Estimators for Weibull Distribution Parameters: Comprehensive Comparative Study for Weibull Distribution
}

\author{
Sahar Sadani $^{\dagger}$, Kamel Abdollahnezhad ${ }^{\dagger, *}$, Mahdi Teimouri ${ }^{\ddagger}$ \\ and Vahid Ranjbar ${ }^{\dagger}$ \\ $\dagger$ Golestan University \\ $\ddagger$ Gonbad Kavous University
}

Received: 7/20/2019 Approved: 10/7/2020

\begin{abstract}
In this paper we focus on two topics. Firstly, we propose $U$-statistics for the Weibull distribution parameters. The consistency and asymptotically normality of the introduced $U$-statistics are proved theoretically and by simulations. Several of methods have been proposed for estimating the parameters of Weibull distribution in the literature. These methods include: the generalized least square type 1, the generalized least square type 2 , the $L$-moments, the Logarithmic moments, the maximum likelihood estimation, the method of moments, the percentile method, the weighted least square, and weighted maximum likelihood estimation. Secondly, due to lack of a comprehensive comparison between the Weibull distribution parameters estimators, a comprehensive comparison study is made between our proposed $U$-statistics and above nine estimators. In our knowledge, this work is the most comprehensive comparison study for the estimators for the Weibull distribution. Based on simulations, it turns out that different estimators may appeal for different range of the parameters. So, practitioners are allowed to chose the best estimator that is suggested by the goodness-of-fit criteria.
\end{abstract}

Keywords. Generalized least square; $L$-moment; logarithmic moment;

\footnotetext{
* Corresponding author
} 
maximum likelihood estimator; $U$-statistic; Weibull distribution; weighted least square; weighted maximum likelihood.

MSC 2010: 62F03, 62F30.

\section{Introduction}

The Weibull distribution is one of the most commonly used distributions with a wide range of applications in some study fields such as: chemical engineering (Chiang et al. (2004), Kuo-Chao et al. (2009), and Wood et al. (2005)), ecology Stankova and Zlatanov (2010), electrical engineering (Genc et al. (2005) and Pascual (2006)), food industry Corzo et al. (2008), mechanical engineering (Raghunathan et al. (2002) and Lavanya et al. (2016)), telecommunications (Surendran et al. (2014) and Buller et al. (2013)), wireless communications Noga and Palczynska (2007), economic (Nadarajah and Kotz (2006) and Diaconu (2009)), civil engineering (Muraleedharan et al. (2007) and Arenas et al. (2010)), and seismology Hasumi et al. (2009). For further details on applications of the Weibull distribution, we refer the readers to Meeker and Escobar (1998), Murthy et al. (2004), and Dodson (2006). However, a comprehensive study has not been performed to compare the estimators. All comparative studies, to the best of our knowledge, have been devoted to compare the performance of the MLE with the estimators of another class. For example, Kanter (2015) made a comparison between least square estimators and the MLEs. The bias of the MLE for the Weibull distribution has been studied by Ross (1996), Watkins (1996) and Montanari et al. (1997). Seki and Yokoyama (1996) made a comparison between the MLE and a bootstrap estimator. Zhang et al. (2007) compared the estimation methods based on the Weibull probability plot. Gebizlioglu et al. (2011) studied different estimation methods of two-parameter Weibull distribution parameters. We also refer the readers to Gebizlioglu et al. (2011), Genschel and Meeker (2010), Hossain and Zimmer (2003), Mohan et al. (2013), Teimouri et al. (2013), and references therein. This is while estimators may have different appeals to different users. For example, the maximum likelihood estimator (MLE) that has attractive properties is biased and has not closed-form expression. This is while the practitioners from some fields may looking for an estimator that is unbiased or has closed-form expression. Also, user may prefer to use an estimator 
which works satisfactorily with sample of small size. Hence, a comparative study is needed to compare the performance of the known estimators under different situations. In this paper, we perform a comprehensive comparison study between ten class of estimators including: the generalized least square type 1 (GLS1), the generalized least square type 2 (GLS2), the $L$-moments (LM), the Logarithmic moments (MLM), the maximum likelihood estimation (MLE), the method of moments (MM), the percentile method (PM), the $U$-statistic, the weighted least square (WLS), and weighted maximum likelihood estimation (WMLE).

The structure of the paper is as follows. In Section 2, we derive $U$-statistic for the shape and scale parameters of the Weibull distribution. The known estimation methods are reviewed briefly in Section 3. Some comparisons will be made in Section 4 through simulation between proposed estimator and known methods in the literature. Furthermore, a real data application is given in this section. We conclude the paper in Section 5 .

\section{$2 U$-Statistics for the Weibull Distribution Pa- rameters}

The probability density function (pdf) and cumulative distribution function (cdf) of two-parameter Weibull distribution are, respectively, given by (Nelson, 1982; Johnson et al., 1994; Dodson, 2006):

$$
\begin{aligned}
& f_{X}(x)=\frac{\alpha}{\beta}\left(\frac{x}{\beta}\right)^{\alpha-1} \exp \left\{-\left(\frac{x}{\beta}\right)^{\alpha}\right\}, \\
& F_{X}(x)=1-\exp \left\{-\left(\frac{x}{\beta}\right)^{\alpha}\right\},
\end{aligned}
$$

for $x>0, \alpha>0$ and $\beta>0$. Here, $\alpha$ and $\beta$ are known as the shape and scale parameters. In the following we give $U$-statistics for the shape and scale parameters of the Weibull distribution. For this, a lemma given by the following is necessary. Hereafter, we write $\mathcal{W}(\alpha, \beta)$ to denote a Weibull distribution with pdf given in (1).

Lemma 1. Suppose $X_{1}, X_{2} \stackrel{i i d}{\sim} \mathcal{W}(\alpha, \beta)$. Then

$$
\min \left\{X_{1}, X_{2}\right\} \stackrel{d}{=} 2^{-\frac{1}{\alpha}} X_{1}
$$


Theorem 1. Let $x_{1}, x_{2}, \ldots, x_{n}$ be observations from a random sample of size $n$ from Weibull distribution with the parameters $\alpha$ and $\beta$ given by (1). For $k=1,2$ let

$$
U_{k}=\left(\begin{array}{l}
n \\
2
\end{array}\right)^{-1} \sum_{1 \leq i<j \leq n} H_{k}\left(x_{i}, x_{j}\right)
$$

where

$$
H_{1}\left(x_{i}, x_{j}\right)=\frac{\log x_{i}+\log x_{j}}{2 \log 2}-\frac{\log \min \left\{x_{i}, x_{j}\right\}}{\log 2}
$$

and

$$
H_{2}\left(x_{i}, x_{j}\right)=\left(1+\frac{\varphi(1)}{\log 2}\right) \frac{\log x_{i}+\log x_{j}}{2}-\frac{\varphi(1)}{\log 2} \log \min \left\{x_{i}, x_{j}\right\},
$$

with $\varphi(1)=-0.57721566$. Then $U_{1}$ and $U_{2}$ are $U$-statistics for $1 / \alpha$ and $\log (\beta)$, respectively.

Proof: First by applying log-transformation to the both sides of (3), we have

$$
\frac{1}{\alpha}=\frac{\log X_{1}-\log \min \left\{X_{1}, X_{2}\right\}}{\log 2} .
$$

Although equating the constant $1 / \alpha$ to a random variable in equation (4) might actually be vague, but that is well-recognized procedure in the literature of the $U$-statistic to extract the kernel. Now, the right-hand side of (4) can be used to construct a symmetric kernel as

$$
H_{1}\left(x_{1}, x_{2}\right)=\frac{\log x_{1}+\log x_{2}}{2 \log 2}-\frac{\log \min \left\{x_{1}, x_{2}\right\}}{\log 2} .
$$

It is easy to see that $\mathrm{E}\left(H_{1}\left(X_{1}, X_{2}\right)\right)=1 / \alpha$. To guarantee the asymptotic normality of the introduced $U$-statistics for $1 / \alpha$ with kernel (5), it is necessary to show that

$$
\operatorname{Var}\left(E\left(H_{1}\left(X_{1}, X_{2}\right) \mid X_{1}\right)\right)<\infty
$$

For this, it suffices to show that $\operatorname{Var}\left(H_{1}\left(X_{1}, X_{2}\right)\right)$ is finite. To begin, we note that $X, X_{1}, X_{2}$ are $i i d$ random variables from $\mathcal{W}(\alpha, \beta)$. 
Since $\min \left\{X_{1}, X_{2}\right\} \stackrel{d}{=} 2^{-\frac{1}{\alpha}} X_{1}$, it follows that

$$
\operatorname{Var}(\log X)=\operatorname{Var}\left(\log \min \left\{X_{1}, X_{2}\right\}\right) .
$$

Here, elementary statistical manipulations reveal that if we define $X=$ $\log \min \left\{X_{1}, X_{2}\right\}, Y=\log X_{1}$, and $Z=\log X_{1}+\log X_{2}$, then

$$
\operatorname{Cov}\left(\log \min \left\{X_{1}, X_{2}\right\}, \log X_{1}+\log X_{2}\right)=\operatorname{Cov}\left(\log X_{1}, \log X_{1}+\log X_{2}\right) \text {. }
$$

Now, using (6), we can write

$$
\begin{aligned}
\operatorname{Var}\left(H_{1}\left(X_{1}, X_{2}\right)\right)= & \frac{\operatorname{Var} \log X}{\log ^{2} 2}+\frac{\operatorname{Var} \log X}{2 \log ^{2} 2} \\
& -\frac{\operatorname{Cov}\left(\log \min \left\{X_{1}, X_{2}\right\}, \log X_{1}+\log X_{2}\right)}{\log ^{2} 2} .
\end{aligned}
$$

Applying property (7) to the right-hand side of (8), we obtain

$$
\operatorname{Var}\left(H_{1}\left(X_{1}, X_{2}\right)\right) \leq \frac{\operatorname{Var}(\log X)}{2 \log ^{2} 2} .
$$

It is easy to check that $\operatorname{Var}(\log X)=\psi(1,1) / \alpha^{2}$ where $\psi(n, x)=\partial^{n} \varphi(x) / \partial x^{n}$ and $\varphi(x)=\partial \log \Gamma(x) / \partial x$. The asymptotic normality of $U_{\alpha}$ follows since

$$
\operatorname{Var}\left(H_{1}\left(X_{1}, X_{2}\right)\right) \leq \frac{\psi(1,1)}{2 \alpha^{2} \log ^{2} 2}<\infty
$$

On the other hand, it is not hard to check that $\mathrm{E}(\log X)=\log \beta+\frac{\varphi(1)}{\alpha}$ where $\varphi(1)=-0.5772157$. Define $H_{2}\left(x_{1}, x_{2}\right)$ as

$$
\begin{aligned}
H_{2}\left(x_{1}, x_{2}\right) & =\frac{\log x_{1}+\log x_{2}}{2}-\varphi(1) H_{1}\left(x_{1}, x_{2}\right) \\
& =\frac{\log x_{1}+\log x_{2}}{2}\left(1-\frac{\varphi(1)}{\log 2}\right)+\frac{\varphi(1)}{\log 2} \log \min \left\{x_{1}, x_{2}\right\} .
\end{aligned}
$$

It is easy to see that $\mathrm{E}\left(H_{1}\left(X_{1}, X_{2}\right)\right)=\log \beta$. Asymptotic normality of the introduced $U$-statistics for $\log \beta$ with kernel (9) holds if we prove $\operatorname{Var}\left(E\left(H_{2}\right.\right.$ $\left.\left.\left(X_{1}, X_{2}\right) \mid X_{1}\right)\right)<\infty$ or equivalently $\operatorname{Var}\left(H_{1}\left(X_{1}, X_{2}\right)\right)<\infty$. We eliminate the proof since kernels $H_{1}\left(x_{1}, x_{2}\right)$ and $H_{2}\left(x_{1}, x_{2}\right)$ have similar structure. 
Remark 1. Estimators given in Theorem 1 are $U$-statistics for $1 / \alpha$ and $\log \beta$, respectively. Estimators of $\alpha$ and $\beta$ are easily obtained using inverse and exponential transforms, respectively.

\section{Known Estimators for the Weibull Distribution}

Here, we review briefly almost all of known estimation methods for the Weibull distribution.

\subsection{Maximum Likelihood Estimation (MLE)}

There is no closed-form expression for MLEs of the Weibull distribution parameters. It is asymptotically normal and efficient for large sample sizes. Many attempts have been made to compute or modify the MLEs of the Weibull distribution parameters. Cohen and Whitten (1982) considered a modified MLE involving complicated numerical computations. Dodson (2006) derived the MLE for the shape parameter graphically. The MLE of the shape parameter is computed as the root of the equation, see Norman et al. (1994)

$$
\frac{n}{\alpha}-\sum_{i=1}^{n} \log x_{i}-n \log \beta+\sum_{i=1}^{n}\left(\frac{x_{i}}{\beta}\right)^{\alpha} \log \left(\frac{x_{i}}{\beta}\right),
$$

and the MLE of the scale parameter is given by

$$
\widehat{\beta}_{M L E}=\left(\frac{\sum_{i=1}^{n} x_{i}^{\alpha}}{n}\right)^{\frac{1}{\alpha}} .
$$

It can be seen that $\widehat{\beta}_{M L E}$ depends on $\alpha$ and also that $\widehat{\alpha}_{M L E}$ must be computed numerically.

\subsection{Weighted Maximum Likelihood (WMLE)}

It is known that MLEs are generally biased. To reduce the bias rate in the case of the Weibull distribution, the weighted maximum likelihood estimators (WMLE) have been proposed in Jacquelin (1993). Suppose $x_{1}, x_{2}, \ldots, x_{n}$ is a random sample from cdf given in (2), then the WMLEs of the shape and 
scale parameters are given by

$$
\begin{aligned}
& \hat{\alpha}_{W M L E}=\arg \min _{\alpha}\left(\frac{W_{2}}{\alpha}+\frac{1}{n} \log x_{i}-\frac{\sum_{i=1}^{n} x_{i}^{\alpha} \log x_{i}}{\sum_{i=1}^{n} x_{i}^{\alpha}}\right)^{2} \\
& \hat{\beta}_{W M L E}=\left(\frac{1}{n W_{1}} \sum_{i=1}^{n} x_{i}^{\alpha}\right)^{\frac{1}{\alpha}}
\end{aligned}
$$

where the weights $W_{1}$ and $W_{2}$ are given by

$$
\begin{gathered}
W_{1}=-\frac{1}{n} \sum_{i=1}^{n} \log \left(1-F\left(X_{i}\right)\right), \\
W_{2}=\frac{\sum_{i=1}^{n} \log \left(1-F\left(X_{i}\right)\right) \log \left(-\log \left(1-F\left(X_{i}\right)\right)\right)}{\sum_{i=1}^{n} \log \left(1-F\left(X_{i}\right)\right)}-\frac{1}{n} \sum_{i=1}^{n} \log \left(-\log \left(1-F\left(X_{i}\right)\right)\right) .
\end{gathered}
$$

Although the sampling distribution of the $W_{1}$ is gamma with shape parameter $n$ and scale parameter $1 / n$, but the sampling distribution of the $W_{2}$ is not known. In practice, both of random variables $W_{1}$ and $W_{2}$ are replaced by their central quantities such as mean, median, or geometric mean. Here, we use the median of $W_{1}$ and $W_{2}$ since they yield the best performance, see Cousineau (2009). For this, in a comprehensive Monte Carlo simulation, we derive the median of $W_{1}$ and $W_{2}$ for different levels of $\alpha$ (from 0.5 to 5 by 0.2 ) and small sample size $n$ (including $5,10,15,30,50,100, \ldots, 200)$. We note that as $n$ tends to $\infty$, both WMLE and MLE approaches give the same results.

\subsection{Generalized and Weighted Least Square (GLS and WLS)}

The parameter estimation using least square approach is common in the statistical literature. For Pareto, log-logistic and Weibull distributions we refer the readers to Kantar (2015), Hung (2001), Lu et al. (2004), Zhang et al. (2008), Van Zyl and Schall (2012), and Zhang et al. (2007). Suppose $x_{(1)} \leq x_{(2)} \leq \cdots \leq x_{(n)}$ are the ordered realizations from Weibull distribution with pdf given in (2). We can see that the following regression model holds.

$$
y_{(i)}=\log \beta+\frac{1}{\alpha} \log \left(-\log \left(1-F\left(x_{(i)}\right)\right)\right)
$$

for $i=1, \ldots, n$ where $y_{(i)}=\log x_{(i)}$. The quantity $F\left(x_{(i)}\right)$, in the right-hand 
side of regression model (10), is replaced by $\frac{i}{n+1}$ or $\frac{i-0.3}{n+0.4}$, see Tiryakioglu and Hudak (2007), Van Zyl and Schall (2012), and Kantar and Yildirim (2015). Since the sample $x_{(i)}$ is ordered, the dependent variable $y_{(i)}$ is also ordered. Therefore the variance of dependent variable is not of the form $\sigma^{2} \mathrm{I}$, see Kantar (2015). To tackle this issue the generalized least square (GLS) technique is proposed, see Engeman and Keefe (1982). The GLS estimate, i.e., $\hat{\boldsymbol{\beta}}_{G L S 1}=(\log \hat{\beta}, 1 / \hat{\alpha})^{T}$ is given by

$$
\hat{\boldsymbol{\beta}}_{G L S 1}=\left(X^{T} V^{-1} X\right) X^{T} V^{-1} Y,
$$

where $Y=\left(\log x_{(1)}, \log x_{(2)}, \ldots, \log x_{(n)}\right)^{T}$,

$$
X=\left(\begin{array}{cc}
1 & \log \left(-\log \left(1-\hat{F}\left(x_{(1)}\right)\right)\right) \\
\vdots & \vdots \\
1 & \log \left(-\log \left(1-\hat{F}\left(x_{(n)}\right)\right)\right)
\end{array}\right)
$$

and

$$
V=\left(\begin{array}{ccc}
v_{11} & \ldots & v_{1 n} \\
\vdots & \vdots & \vdots \\
v_{n 1} & \ldots & v_{n n}
\end{array}\right)
$$

for

$v_{i j}=\frac{i}{(n+1-i)} \frac{1}{\log (n+1-i)-\log (n+1)} \frac{1}{\log (n+1-j)-\log (n+1)} ; \quad i \leq j$.

The second type of GLS estimate, i.e.,

$$
\hat{\boldsymbol{\beta}}_{G L S 2}=\left(Z^{T} V^{-1} X\right) Z^{T} V^{-1} Y,
$$

can be constructed if we replace $X$ with $Z$ as

$$
Z=\left(\begin{array}{ccc}
1 & \log \left(-\log \left(1-\hat{F}\left(x_{(1)}\right)\right)\right)-0.5-\frac{\log \left(1-\hat{F}\left(x_{(1)}\right)\right)}{\left(\left(1-\hat{F}\left(x_{(1)}\right)\right) \log \left(1-\hat{F}\left(x_{(1)}\right)\right)\right)^{2}} \\
\vdots & \vdots & \log \left(1-\hat{F}\left(x_{(n)}\right)\right) \\
1 & \log \left(-\log \left(1-\hat{F}\left(x_{(n)}\right)\right)\right)-0.5-\frac{\left.\left.\hat{F}\left(x_{(n)}\right)\right)\right)^{2}}{\left(( 1 - \hat { F } ( x _ { ( n ) } ) ) \operatorname { l o g } \left(1-{ }_{0}\right.\right.}
\end{array}\right) .
$$


We note that $\hat{\boldsymbol{\beta}}_{G L S 2}=(\log \hat{\beta}, 1 / \hat{\alpha})^{T}$ and $\hat{F}\left(x_{(i)}\right)=\frac{i}{n+1}$. The weighted least square (WLS) estimate are also given by

$$
\hat{\boldsymbol{\beta}}_{W L S}=\left(X^{T} W^{-1} X\right) X^{T} W^{-1} Y,
$$

where $\hat{\boldsymbol{\beta}}_{W L S}=(\log \hat{\beta}, 1 / \hat{\alpha})^{T}$ and $W$ is a diagonal matrix whose entries are $v_{11}, \ldots, v_{n n}$, see Kantar (2015).

\section{$3.4 \quad L$-moment (LM)}

The $L$-moments have their origin in works by Hosking (1990) and Elamir and Seheult (2003). By equating the sample $L$-moment to the population counterpart gives the $L$-moment estimate. The $r$-th $L$-moment of Weibull distribution with pdf (1) is given by:

$\mu_{r}^{L}=\frac{\beta}{r} \Gamma\left(\frac{1}{\alpha}+1\right) \sum_{k=0}^{r-1}(-1)^{k} C_{k}^{r-1}(r-k) C_{r-k}^{r} \sum_{j=0}^{r-k-1} C_{j}^{r-k-1} \frac{(-1)^{j}}{(k+j+1)^{1 / \alpha+1}}$,

where $\alpha>0, \beta>0, r=1,2, \ldots$, and $C_{i}^{n}$ denotes the binomial coefficient $n ! /(i !(n-i) !)$, see Hosking (1990). So the first and the second $L$-moments are given by $\mu_{1}^{L}=\beta \Gamma(1 / \alpha+1)$ and $\mu_{2}^{L}=\beta \Gamma(1 / \alpha+1)\left(1-2^{-1 / \alpha}\right)$, respectively. The first two sample $L$-moments are:

$$
m_{1}^{L}=\frac{1}{n} \sum_{i=1}^{n} X_{i: n}=\bar{X}
$$

and

$$
m_{2}^{L}=\frac{2}{n(n-1)} \sum_{i=1}^{n}(i-1) X_{i: n}-\bar{X}
$$

Now, equating $\mu_{1}^{L}$ and $\mu_{2}^{L}$ with $m_{1}^{L}$ and $m_{2}^{L}$, respectively, the $L$-moments of $\alpha$ and $\beta$ are obtained as:

$$
\widehat{\alpha}_{L M}=-\frac{\ln (2)}{\ln \left(1-m_{2}^{L} / m_{1}^{L}\right)}
$$


and

$$
\widehat{\beta}_{L M}=\frac{m_{1}^{L}}{\Gamma\left(1 / \widehat{\alpha}_{L M}+1\right)} .
$$

\subsection{Method of Logarithmic Moment (MLM)}

The log-moment estimates of the shape and scale parameters of Weibull distribution with cdf (2) are given by (see Wayne (1982), Norman et al. (1994), and Dodson (2006))

$$
\widehat{\alpha}_{M L M}=\sqrt{\frac{\pi^{2}}{6 S^{2}}},
$$

and

$$
\widehat{\beta}_{M L M}=\exp \left\{M_{1}-\psi(1) / \widehat{\alpha}_{M L M}\right\},
$$

where $S^{2}$ and $M_{1}$ are the sample variance and the mean of log-transformed data, respectively. Also $\psi(1)=-0.5772156$. It can be shown that (14) and (15) are both asymptotically unbiased and consistent, see Norman et al. (1994).

\subsection{Percentile Method (PM)}

The quantile of a Weibull distribution with cdf (2) is

$$
x_{p}=\beta[-\ln (1-p)]^{1 / \alpha},
$$

where $0<p<1$, see Norman et al. (1994) and Dodson (2006). Using $p=1-\exp (-1) \cong 0.632$, one can construct percentile-based estimators for $\alpha$ and $\beta$ as

$$
\hat{\alpha}_{P M}=\left(\frac{\ln [-\ln (1-p)]}{\ln \left(x_{p}\right)-\ln \left(x_{0.632}\right)}\right)
$$

and

$$
\hat{\beta}_{P M}=x_{1-\exp (-1)},
$$


respectively, where $0<x_{p}<x_{0.632}$. The suggested values for $p$ are 0.15 (see Wang and Keats (1995)) and 0.31, see (Seki and Yokoyama (1996) and Hassanein (1971)). Statistical tools show that percentile-based estimators are, in general, asymptotically normal and unbiased, see Wayne (1982).

\subsection{Method of Moments (MM)}

Moment-based estimators of a given population are obtained by equating the population moments to their sample counterparts and solving the resulting equations. The moment-based estimators for the Weibull distribution suffers from numerical computations, see Cran (1988). Also, these estimators are not efficient. The $r$-th non-central moment for the Weibull distribution is (Wayne (1982); Norman et al. (1994); Dodson (2006)):

$$
\mu_{r}=\beta^{r} \Gamma(r / \alpha+1) .
$$

Equating the mean and variance $\left(\mu_{1}\right.$ and $\left.\mu_{2}-\mu_{1}^{2}\right)$ with the sample counterparts $\left(\bar{X}\right.$ and $S^{2}$ ), the moment-based estimator of the shape parameter $\widehat{\alpha}_{M M}$, is root of the equation

$$
\frac{\Gamma(1+2 / \alpha)}{\Gamma^{2}(1+1 / \alpha)}+\frac{S^{2}}{\bar{X}}-1=0
$$

and the moment-based estimator of the scale parameter is

$$
\widehat{\beta}_{M M}=\frac{\bar{X}}{\Gamma\left(1 / \widehat{\alpha}_{M M}+1\right)} .
$$

\section{Performance Comparisons}

This section has two parts. In the first part, we compare the performances of estimators introduced in Section 2 and 3 through simulation. Second part devoted to an illustration in which all estimators are applied to a set of real data.

\subsection{Simulation Study}

Here, we perform a Monte Carlo simulation to compare the performance of the $U$-statistic, MLE, WMLE, GLS1, GLS2, WLS, LM, MLM, PM, and MM. For this aim, we compare the bias and root of mean squared error (RMSE). 
For computing the bias we adopt small sizes of sample including 5, 10, 30, and two levels for shape and scale parameters as: $(0.5,0.5),(2.5,0.5),(0.5$, $2.5)$, and $(2.5,2.5)$. The results after computing the bias are given in Tables 1-2. Also the bias of $U$-statistic, MLE, GLS1, GLS2, WLS, and LM are given for large sizes of sample including 1000 and 4000. The corresponding results are given in Tables 3-4 for shape and scale parameters, respectively.

For computing the RMSE, we choose the sample sizes as: 5, 10, 15, 30, 50,100 , and 200. Comparisons are performed for different levels of the shape $(\alpha=0.5,1$, and 2.5$)$ and the scale $(\beta=0.5,2$, and 5$)$ parameters. We used a 7-color scheme to distinguish between competitors through Figures 1-2 as follows. The brown for the $U$-statistic, green for the MLE, purple for the WMLE, dashed red for the GLS1, black for the GLS2, blue for the WLS, dashed purple for the MLM, dotted purple for the PM, solid red for the MM, and yellow solid curve for LM. The results for computing the RMSE are given in Figures 1-2.

\subsubsection{Comparison Results for the Bias}

According to the bias of the shape parameter estimator $\hat{\alpha}$ for small sizes 5 , 10, and 30, the following conclusions can be made from Table 1 .

1. GLS2, WLS, and WMLE give the best performances for $n=5, n=10$, and $n=30$, respectively.

2. WMLE shows the best performance next to the WLS and GLS1.

3. PM gives the worst performance.

4. When $\alpha$ is small (say $\alpha=0.5$ ), the MM gives the worst performance next to the GLS2.

5. When $\alpha$ is large (say $\alpha=2.5$ ) and $n \geq 15$, the GLS2 gives the worst performance.

6. WMLE outperforms the LM.

7. WMLE and $U$-statistic outperform the MLE.

8. U-statistic shows better performance than the MLE, MLM, MM, and PM. 
The following observations can be made from Table 2 for bias of the scale parameter estimator $\hat{\beta}$ for small sizes 5,10 , and 30 .

1. WMLE and MLE give almost the same performances.

2. When $\alpha$ is small (say $\alpha=0.5$ ), the MM gives the worst performance.

3. When $\alpha$ is small, the LM gives the best performance.

4. The GLS2, GLS1, and WLS show the same performances.

5. MLM outperforms GLS2, GLS1, and WLS.

6. When $\alpha$ is large (say $\alpha=2.5$ ), the PM shows the worst performance.

7. The GLS2, GLS1, and WLS outperforms the $U$-statistic for $n=5,10$.

The following observations can be made from Tables 3-4 for bias of the shape parameter estimator $\hat{\beta}$ for large sizes 1000 and 4000 .

1. $U$-statistic gives the best performance for estimating the shape and scale parameters.

2. GLS1 shows the worst performance for estimating the shape and scale parameters.

We note that for bias analysis when sample sizes are large, the MLM, PM, and MM have been eliminated by competitions since the show weak performances. Also, since MLE and WMLE show the same performances, the WMLE has been removed by competitions.

\subsubsection{Comparison Results for RMSE}

The following observations can be made from Figure 1 for RMSE of the shape parameter estimator $\hat{\alpha}$.

1. The PM gives the worst performance.

2. When $n=5$ the GLS2 gives the best performance.

3. When $n=5$ the GLS2 gives the best performance.

4. The WGLS gives the best performance next to the GLS1.

5. The WMLE outperforms the LM and $U$-statistic. 
6. The MLM shows better performance than the MLE for sample size (say $n \leq 10$ ).

The following observations can be made from Figure 2 for RMSE of the scale parameter estimator $\hat{\beta}$.

1. The PM gives the worst performance.

2. When $\alpha$ is small (say $\alpha \leq 0.5$ ), the MM gives the worst performance.

3. When $\alpha$ is not small (say $\alpha \geq 1$ ), the PM gives the worst performance.

4. When $\alpha$ is small (say $\alpha \leq 0.5$ ) and $n \leq 15$, the LM gives the best performance.

\subsection{Real Data Illustration}

Here, we apply all reviewed methods introduced in Sections 2 and 3 to a set of real data involving by lifetimes in years reported by (Glick, 1978, p. 17). Data are shown in Table 5. Moreover, using a bootstrap method by $\mathrm{B}=10000$ iterations the standard errors of estimators computed. To implement these techniques, programs have been written in R environment, see Team (2014). In order to compare the performance of estimators presented in the Section 2 and 3, we employed the Kolmogorov-Smirnov (KS) and Cramer-Von Mises (CVM) distances which are given by

$$
\mathrm{KS}=\max _{\mathbf{1} \leq \boldsymbol{i} \leq \boldsymbol{n}} \max \left\{\frac{i}{n}-F_{X}\left(x_{(i)}\right), F_{X}\left(x_{(i)}\right)-\frac{i-1}{n}\right\},
$$

and

$$
\mathrm{CVM}=\frac{1}{12 n}+\sum_{i=1}^{n}\left[\frac{2 i-1}{2 n}-F_{X}\left(x_{(i)}\right)\right]^{2},
$$

where $n$ is the sample size, $x_{(i)}$; for $i=1 \ldots, n$, is the $i$-th ordered observed value and $F_{X}($.$) is the distribution function of two-parameter Weibull distri-$ bution defined in (2). The following observations can be made from Table 6 .

1. The WLS shows the best performance in the sense of both criteria KS and CVM.

2. The MLM shows the best performance in the sense of CVM criterion next to the WLS. 
3. The PM shows the best performance in the sense of KS criterion next to the WLS.

\section{Conclusion}

We have introduced $U$-statistics for shape and scale parameters of twoparameter Weibull distribution. Asymptotic normality and consistency of the new estimators have been proved. Furthermore, a comprehensive Monte Carlo study have been carried out to compare the performance of the known estimators of the two-parameter Weibull distribution parameters. A pointed out by one of referees since structure of estimators studied in this work depends on both of the shape and scale parameters, so they are statistically dependent. The degree of dependence between estimators of the shape and scale parameters under each method can be considered as a possible future work. On the other hand, different estimators may appeal different users for different levels of sample size and parameters levels, a list of comparisons have been made in the paper for choosing desired estimator. Many facts can be concluded from this study, among them our results are the followings.

- for small sizes of samples the weighted least square (WLS) approach gives the best performance in the sense of bias.

- shape estimator based on method of weighted least square (WLS) gives the best performance root of mean squared error (RMSE).

- shape estimator based on method of percentile gives the worst performance in terms of RMSE.

- shape and scale estimators based on $U$-statistic show the best performances in the sense of bias for large sample sizes.

- shape and scale estimators based on generalized least square type-I (GLS1) approach show the worst performances in the sense of bias for large sample sizes. 
Table 1. Bias of shape parameter estimators for samples of small size.

\begin{tabular}{|c|c|c|c|c|}
\hline & \multicolumn{4}{|c|}{$\mathrm{n}=5$} \\
\hline & \multicolumn{4}{|c|}{ parameters level } \\
\hline Method & $(\alpha=0.5, \beta=0.5)$ & $(\alpha=0.5, \beta=2.5)$ & $(\alpha=2.5, \beta=0.5)$ & $(\alpha=2.5, \beta=2.5)$ \\
\hline$U$-Statistic & 0.346 & 0.383 & 1.890 & 1.415 \\
\hline MLE & 0.422 & 0.473 & 2.453 & 1.813 \\
\hline WMLE & 0.299 & 0.340 & 1.764 & 1.309 \\
\hline GLS1 & 0.266 & 0.292 & 1.420 & 1.106 \\
\hline GLS2 & 0.249 & 0.255 & 1.261 & 1.025 \\
\hline WLS & 0.252 & 0.283 & 1.449 & 1.082 \\
\hline LM & 0.331 & 0.359 & 1.798 & 1.353 \\
\hline MLM & 0.405 & 0.443 & 2.203 & 1.645 \\
\hline PM & 1.199 & 1.699 & 8.597 & 7.132 \\
\hline \multirow[t]{3}{*}{ MM } & 0.450 & 0.476 & 1.971 & 1.452 \\
\hline & \multicolumn{4}{|c|}{$\mathrm{n}=10$} \\
\hline & \multicolumn{4}{|c|}{ parameters level } \\
\hline Method & $(\alpha=0.5, \beta=0.5)$ & $(\alpha=0.5, \beta=2.5)$ & $(\alpha=2.5, \beta=0.5)$ & $(\alpha=2.5, \beta=2.5)$ \\
\hline$U$-Statistic & 0.181 & 0.189 & 0.865 & 0.713 \\
\hline MLE & 0.193 & 0.195 & 0.914 & 0.776 \\
\hline WMLE & 0.161 & 0.162 & 0.756 & 0.636 \\
\hline GLS1 & 0.159 & 0.164 & 0.765 & 0.608 \\
\hline GLS2 & 0.196 & 0.195 & 0.966 & 0.749 \\
\hline WLS & 0.148 & 0.146 & 0.697 & 0.572 \\
\hline LM & 0.193 & 0.191 & 0.781 & 0.649 \\
\hline MLM & 0.205 & 0.216 & 0.999 & 0.817 \\
\hline PM & 0.546 & 0.553 & 2.491 & 2.334 \\
\hline \multirow[t]{3}{*}{ MM } & 0.279 & 0.278 & 0.806 & 0.664 \\
\hline & \multicolumn{4}{|c|}{$\mathrm{n}=30$} \\
\hline & \multicolumn{4}{|c|}{ parameters level } \\
\hline Method & $(\alpha=0.5, \beta=0.5)$ & $(\alpha=0.5, \beta=2.5)$ & $(\alpha=2.5, \beta=0.5)$ & $(\alpha=2.5, \beta=2.5)$ \\
\hline$U$-Statistic & 0.071 & 0.074 & 0.383 & 0.301 \\
\hline MLE & 0.078 & 0.079 & 0.410 & 0.332 \\
\hline WMLE & 0.074 & 0.073 & 0.379 & 0.309 \\
\hline GLS1 & 0.085 & 0.084 & 0.425 & 0.342 \\
\hline GLS2 & 0.121 & 0.119 & 0.588 & 0.468 \\
\hline WLS & 0.077 & 0.076 & 0.385 & 0.311 \\
\hline LM & 0.099 & 0.110 & 0.393 & 0.315 \\
\hline MLM & 0.095 & 0.096 & 0.493 & 0.400 \\
\hline PM & 0.184 & 0.191 & 1.045 & 0.867 \\
\hline MM & 0.150 & 0.149 & 0.386 & 0.313 \\
\hline
\end{tabular}


Table 2. Bias of scale parameter estimators for samples of small size.

\begin{tabular}{|c|c|c|c|c|}
\hline & \multicolumn{4}{|c|}{$\mathrm{n}=5$} \\
\hline & \multicolumn{4}{|c|}{ parameters level } \\
\hline Method & $(\alpha=0.5, \beta=0.5)$ & $(\alpha=0.5, \beta=2.5)$ & $(\alpha=2.5, \beta=0.5)$ & $(\alpha=2.5, \beta=2.5)$ \\
\hline$U$-Statistic & 0.816 & 2.717 & 0.094 & 0.481 \\
\hline MLE & 0.674 & 2.318 & 0.091 & 0.460 \\
\hline WMLE & 0.673 & 2.315 & 0.091 & 0.459 \\
\hline GLS1 & 0.809 & 2.686 & 0.094 & 0.481 \\
\hline GLS2 & 0.795 & 2.641 & 0.093 & 0.477 \\
\hline WLS & 0.806 & 2.670 & 0.094 & 0.479 \\
\hline LM & 0.544 & 1.909 & 0.093 & 0.469 \\
\hline MLM & 0.723 & 2.431 & 0.092 & 0.468 \\
\hline $\mathrm{PM}$ & 0.903 & 2.943 & 0.099 & 0.508 \\
\hline \multirow[t]{3}{*}{ MM } & 0.927 & 2.983 & 0.092 & 0.463 \\
\hline & \multicolumn{4}{|c|}{$\mathrm{n}=10$} \\
\hline & \multicolumn{4}{|c|}{ parameters level } \\
\hline Method & $(\alpha=0.5, \beta=0.5)$ & $(\alpha=0.5, \beta=2.5)$ & $(\alpha=2.5, \beta=0.5)$ & $(\alpha=2.5, \beta=2.5)$ \\
\hline$U$-Statistic & 0.424 & 1.752 & 0.067 & 0.337 \\
\hline MLE & 0.389 & 1.608 & 0.066 & 0.329 \\
\hline WMLE & 0.387 & 1.596 & 0.066 & 0.329 \\
\hline GLS1 & 0.385 & 1.652 & 0.067 & 0.337 \\
\hline GLS2 & 0.421 & 1.744 & 0.067 & 0.336 \\
\hline WLS & 0.423 & 1.752 & 0.067 & 0.338 \\
\hline $\mathrm{LM}$ & 0.349 & 1.455 & 0.067 & 0.332 \\
\hline MLM & 0.409 & 1.663 & 0.067 & 0.335 \\
\hline $\mathrm{PM}$ & 0.485 & 1.948 & 0.077 & 0.393 \\
\hline \multirow[t]{3}{*}{ MM } & 0.513 & 2.129 & 0.066 & 0.331 \\
\hline & \multicolumn{4}{|c|}{$\mathrm{n}=30$} \\
\hline & \multicolumn{4}{|c|}{ parameters level } \\
\hline Method & $(\alpha=0.5, \beta=0.5)$ & $(\alpha=0.5, \beta=2.5)$ & $(\alpha=2.5, \beta=0.5)$ & $(\alpha=2.5, \beta=2.5)$ \\
\hline$U$-Statistic & 0.215 & 0.866 & 0.038 & 0.186 \\
\hline MLE & 0.207 & 0.841 & 0.037 & 0.187 \\
\hline WMLE & 0.207 & 0.842 & 0.037 & 0.188 \\
\hline GLS1 & 0.214 & 0.869 & 0.038 & 0.191 \\
\hline GLS2 & 0.215 & 0.869 & 0.038 & 0.191 \\
\hline WLS & 0.215 & 0.874 & 0.038 & 0.191 \\
\hline LM & 0.201 & 0.830 & 0.037 & 0.188 \\
\hline MLM & 0.218 & 0.857 & 0.038 & 0.195 \\
\hline $\mathrm{PM}$ & 0.257 & 1.060 & 0.045 & 0.227 \\
\hline MM & 0.284 & 1.110 & 0.037 & 0.202 \\
\hline
\end{tabular}

J. Statist. Res. Iran 16 (2019): 33-57 
Table 3. Bias of shape parameter estimators for samples of large size.

\begin{tabular}{lcccc}
\hline \multicolumn{5}{c}{$\mathrm{n}=1000$} \\
\hline Method & $(\alpha=0.5, \beta=0.5)$ & $(\alpha=0.5, \beta=2.5)$ & $(\alpha=2.5, \beta=0.5)$ & $(\alpha=2.5, \beta=2.5)$ \\
\hline GLS1 & 0.005193 & 0.003696 & 0.018966 & 0.008460 \\
WLS & 0.005025 & 0.003351 & 0.017895 & 0.006642 \\
GLS2 & -0.005167 & -0.002230 & -0.016367 & -0.007867 \\
MLE & -0.004958 & -0.002026 & -0.017401 & -0.005958 \\
LM & 0.004229 & 0.002091 & 0.016958 & 0.005620 \\
U-Statistic & 0.003600 & 0.001065 & 0.013052 & 0.003600 \\
\hline \multicolumn{5}{c}{ parameters level } \\
\hline Method & $(\alpha=0.5, \beta=0.5)$ & $(\alpha=0.5, \beta=2.5)$ & $(\alpha=2.5, \beta=0.5)$ & $(\alpha=2.5, \beta=2.5)$ \\
\hline GLS1 & 0.002892 & $0.976 \mathrm{e}-03$ & 0.009939 & 0.003492 \\
WLS & 0.002632 & $0.764 \mathrm{e}-03$ & 0.009614 & 0.002632 \\
GLS2 & -0.002260 & $-6.088 \mathrm{e}-04$ & -0.009609 & -0.002260 \\
MLE & -0.002372 & $-9.705 \mathrm{e}-04$ & -0.009305 & -0.002372 \\
LM & 0.001498 & $-7.164 \mathrm{e}-04$ & -0.007535 & 0.002498 \\
U-Statistic & 0.001253 & $2.018 \mathrm{e}-04$ & -0.004859 & 0.001253 \\
\hline
\end{tabular}

Table 4. Bias of scale parameter estimators for samples of large size.

\begin{tabular}{lcccc}
\hline \multicolumn{5}{c}{$\mathrm{n}=1000$} \\
\hline Method & $(\alpha=0.5, \beta=0.5)$ & $(\alpha=0.5, \beta=2.5)$ & $(\alpha=2.5, \beta=0.5)$ & $(\alpha=2.5, \beta=2.5)$ \\
\hline GLS1 & 0.006450 & 0.013542 & 0.006873 & -0.014509 \\
WLS & 0.004087 & 0.011946 & 0.006442 & 0.013873 \\
GLS2 & 0.004891 & 0.011840 & 0.005900 & 0.011489 \\
MLE & 0.005883 & 0.011700 & -0.005236 & 0.010988 \\
LM & 0.006123 & 0.010598 & -0.006468 & 0.012319 \\
U-Statistic & 0.003323 & 0.007526 & 0.005378 & 0.009353 \\
\hline & & \multicolumn{4}{c}{$\mathrm{n}=4000$} & \\
\hline \multicolumn{5}{c}{ parameters level } \\
\hline Method & $(\alpha=0.5, \beta=0.5)$ & $(\alpha=0.5, \beta=2.5)$ & $(\alpha=2.5, \beta=0.5)$ & $(\alpha=2.5, \beta=2.5)$ \\
\hline GLS1 & 0.001854 & 0.005237 & $1.968 \mathrm{e}-03$ & 0.002354 \\
WLS & 0.001263 & 0.003971 & $1.879 \mathrm{e}-03$ & 0.001263 \\
GLS2 & 0.001495 & 0.003879 & $1.807 \mathrm{e}-03$ & 0.001495 \\
MLE & 0.001382 & 0.004028 & $1.748 \mathrm{e}-03$ & 0.001382 \\
LM & 0.001545 & -0.003890 & $1.651 \mathrm{e}-03$ & 0.001845 \\
U-Statistic & 0.000552 & -0.002518 & $1.029 \mathrm{e}-03$ & 0.000552 \\
\hline
\end{tabular}


Table 5. Lifetime data (in year)

\begin{tabular}{llllllllllll}
\hline 30.20 & 36.55 & 25.11 & 39.35 & 27.57 & 25.91 & 31.50 & 29.24 & 18.39 & 16.65 & 21.85 & 24.88 \\
31.61 & 18.74 & 19.63 & 28.98 & 11.10 & 21.66 & 22.41 & 26.04 & 25.07 & 23.48 & 28.21 & 25.21 \\
25.12 & 27.76 & 23.47 & 23.51 & 24.39 & 21.93 & 37.63 & 20.32 & 28.17 & 24.66 & 30.13 & 21.42 \\
17.21 & 19.98 & 33.09 & 16.04 & 17.96 & 19.57 & 22.91 & 25.69 & 23.47 & 16.91 & 27.20 & 27.23 \\
\hline
\end{tabular}

Table 6. Estimation results and their standard errors (in parentheses) after fitting twoparameter Weibull distribution to the lifetime data.

\begin{tabular}{lcccc}
\hline & \multicolumn{2}{c}{ Estimated parameters } & \multicolumn{2}{c}{ goodness-of-fit measures } \\
\hline Method & $\hat{\alpha}$ & $\hat{\beta}$ & KS & CVM \\
\hline$U$-statistic & $5.1575(0.6814)$ & $26.8644(0.7876)$ & 0.0934 & 0.0591 \\
MLE & $4.5922(0.5561)$ & $26.9452(0.8952)$ & 0.0920 & 0.0713 \\
WMLE & $4.5141(0.5314)$ & $26.9370(0.9082)$ & 0.0906 & 0.0744 \\
GLS1 & $4.7548(0.6105)$ & $26.9926(0.8685)$ & 0.0971 & 0.0721 \\
GLS2 & $4.3035(0.4892)$ & $26.9788(0.9554)$ & 0.0904 & 0.0926 \\
WLS & $4.7099(0.5658)$ & $26.6979(0.8861)$ & 0.0777 & 0.0482 \\
LM & $4.9512(0.6229)$ & $26.9055(0.8309)$ & 0.0939 & 0.0609 \\
MLM & $5.3119(0.8072)$ & $26.7771(0.7787)$ & 0.0889 & 0.0529 \\
PM & $5.9767(1.6019)$ & $25.8461(0.8132)$ & 0.0867 & 0.0622 \\
MM & $4.9150(0.6140)$ & $26.9169(0.8413)$ & 0.0942 & 0.0621 \\
\hline
\end{tabular}



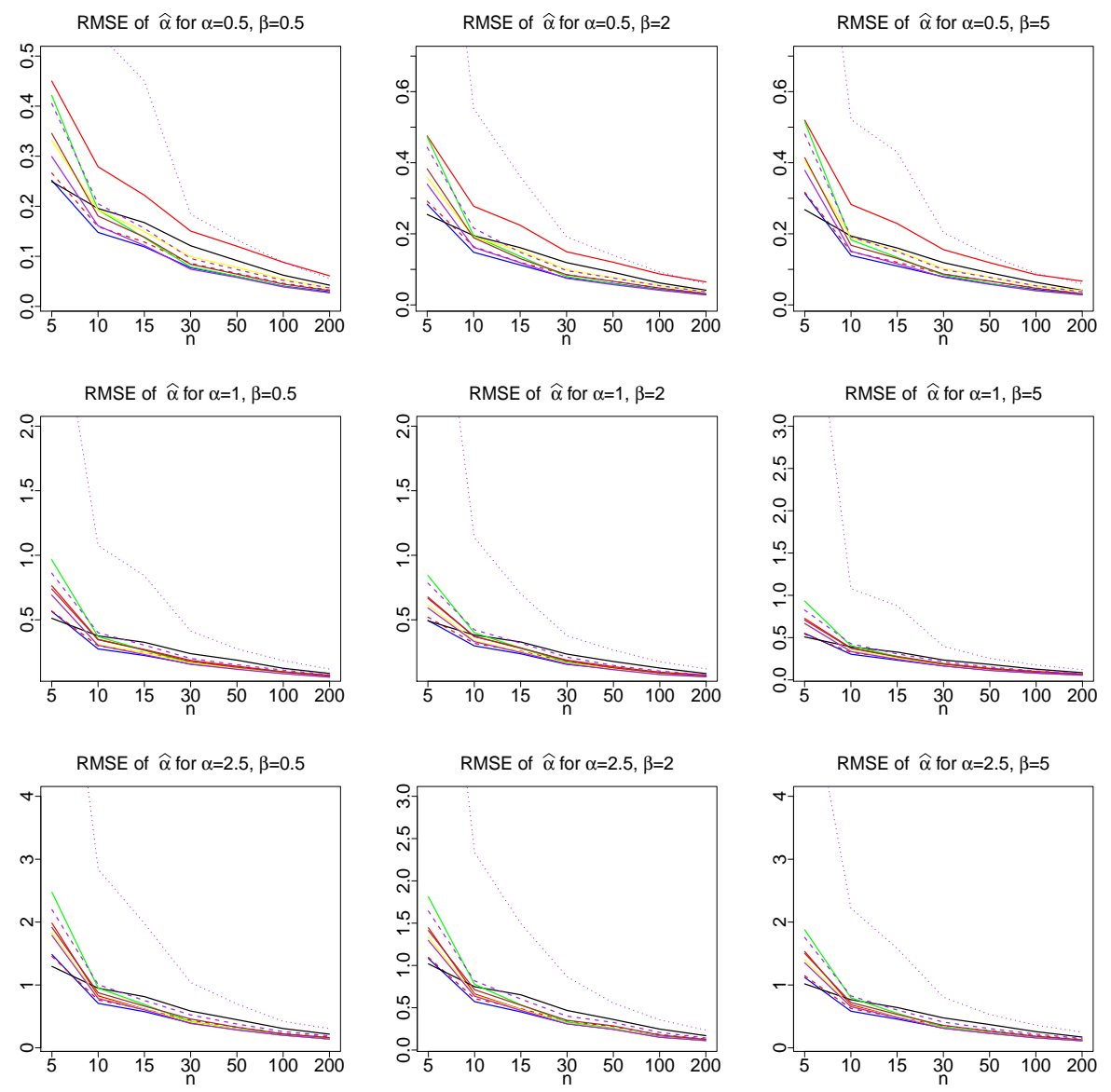

Figure 1. RMSE of the shape parameter estimator, $\hat{\alpha}$ for different levels of $\alpha$ and $\beta$ under small sample size scenario, i.e., $n=5,10,15,30,50,100$, and 200. The used color scheme are: the black solid curve for the GLS2, blue solid curve for the WLS, brown solid curve for the $U$-statistic, green solid curve for the MLE, solid red curve for the MM, dashed red curve for the GLS1, purple solid curve for the WMLE, dotted purple curve for the PM, dashed purple curve for the MLM, and yellow solid curve for LM. 

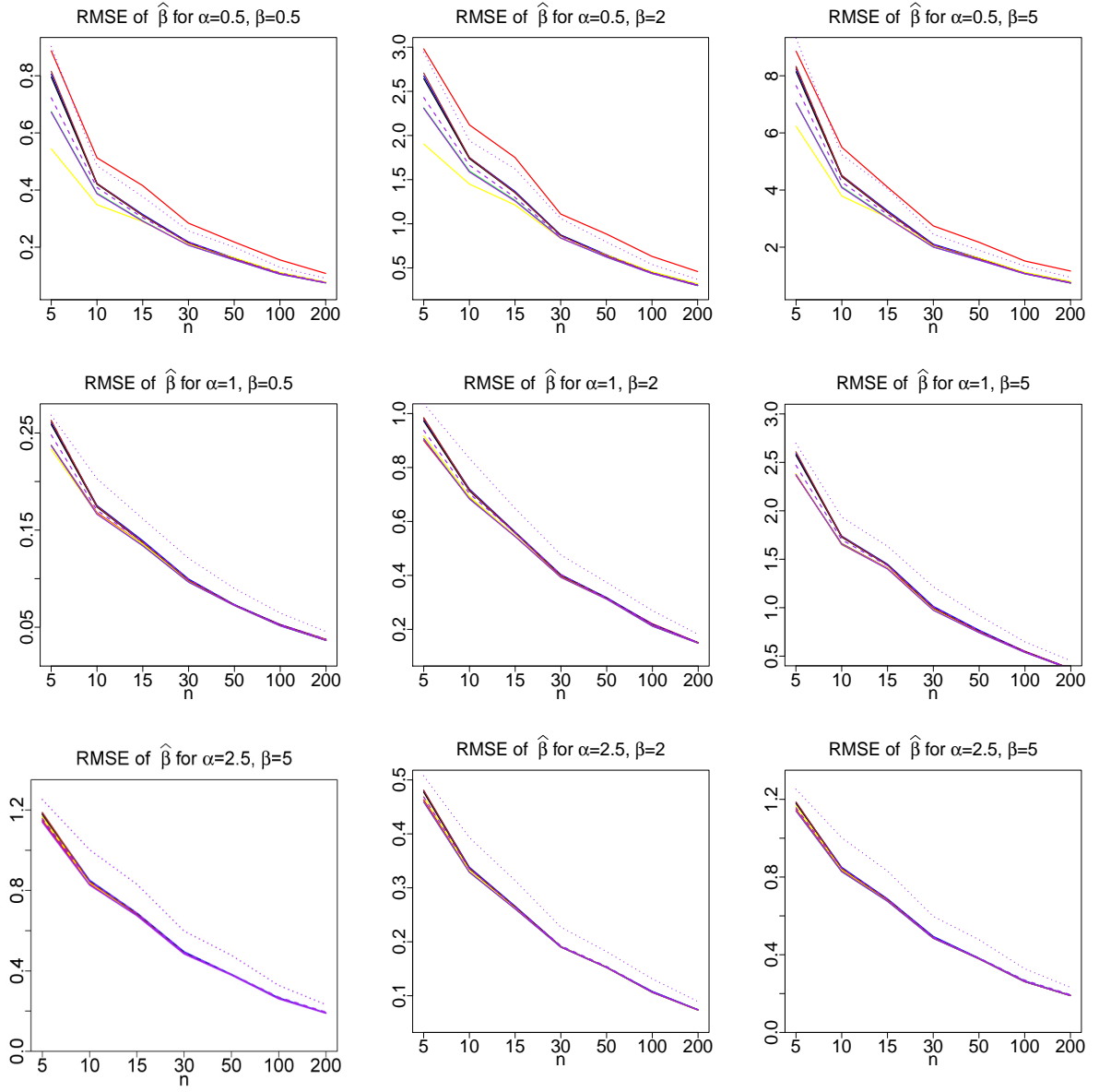

Figure 2. RMSE of the scale parameter estimator, $\hat{\beta}$ for different levels of $\alpha$ and $\beta$ under small sample size scenario, i.e., $n=5,10,15,30,50,100$, and 200 . The used color scheme are: the black solid curve for the GLS2, blue solid curve for the WLS, brown solid curve for the $U$-statistic, green solid curve for the MLE, solid red curve for the MM, dashed red curve for the GLS1, purple solid curve for the WMLE, dotted purple curve for the PM, dashed purple curve for the MLM, and yellow solid curve for LM. 


\section{References}

Arenas, J.M., Narbon, J.J. and Alia, C. (2010). Optimum Adhesive Thickness in Structural Adhesives Joints using Statistical Techniques Based on Weibull Distribution. International Journal of Adhesion and Adhesives, 30, 160-165.

Buller, W., Wilson, B., Van Nieuwstadt, L. and Ebling, J. (2013). Statistical Modelling of Measured Automotive Radar Reections. In Instrumentation and Measurement Technology Conference (I2MTC), 2013 IEEE International, 349-352.

Chiang, Y.J., Shih, C., Lin, C. and Tseng, Y. (2004). Examination of Tyre Rubber Cure by Weibull Distribution Functions. International Journal of Materials and Product Technology, 20, 210-219.

Corzo, O., Bracho, N., Pereira, A. and Vasquez, A. (2008). Weibull Distribution for Modeling Air Drying of Coroba Slices. LWT-Food Science and Technology, 41, 2023-2028.

Cousineau, D. (2009). Nearly Unbiased Estimators for the Three-parameter Weibull Distribution with Greater Efficiency than the Iterative Likelihood Method. British Journal of Mathematical and Statistical Psychology, 62, 167-191.

Cran, G. (1988). Moment Estimators for the 3-parameter Weibull Distribution. IEEE Transac tions on Reliability, 37, 360-363.

Diaconu, A. (2009). Weibull Distribution as a Model for Growth/Decline in Product Sales. Metalurgia International, 14, 184-185.

Dodson, B. (2006). The Weibull Analysis Handbook, ASQ Quality Press.

Engeman, R.M. and Keefe, T.J. (1982). On Generalized Least Squares Estimation of the Weibull Distribution. Communications in Statistics-Theory and Methods, 11, 2181-2193.

Gebizlioglu, O.L., Senoglu, B. and Kantar, Y.M. (2011). Comparison of Certain Value-atrisk Estimation Methods for the Two-parameter Weibull Loss Distribution. Journal of Computational and Applied Mathematics, 235, 3304-3314.

Genc, A., Erisoglu, M., Pekgor, A., Oturanc, G., Hepbasli, A. and Ulgen, K. (2005). Estimation of Wind Power Potential Using Weibull Distribution. Energy Sources, 27, 809-822.

Genschel, U. and Meeker, W.Q. (2010). A Comparison of Maximum Likelihood and Medianrank Regression for Weibull Estimation. Quality Engineering, 22, 236-255.

Glick, N. (1978). Breaking Records and Breaking Boards. The American Mathematical Monthly, 85, 2-26.

Hassanein, K.M. (1971). Percentile Estimators for the Parameters of the Weibull Distribution. Biometrika, 58, 673-676. 
Hasumi, T., Akimoto, T. and Aizawa, Y. (2009). The Weibull Log Weibull Distribution for Interoccurrence Times of Earthquakes. Physica A: Statistical Mechanics and its Applications, 388, 491-498.

Hosking, J.R. (1990). L-moments: Analysis and Estimation of Distributions Using Linear Combinations of Order Statistics. Journal of the Royal Statistical Society. Series B (Methodological), 105-124.

Hossain, A. and Zimmer, W. (2003). Comparison of Estimation Methods for Weibull Parameters: Complete and Censored Samples. Journal of Statistical Computation and Simulation, 73, 145-153.

Hung, W.L. (2001). Weighted Least-squares Estimation of the Shape Parameter of the Weibull Distribution. Quality and Reliability Engineering International, 17, 467-469.

Jacquelin, J. (1993). Generalization of the Method of Maximum Likelihood. IEEE Transactions on Electrical Insulation, 28, 65-72.

Kantar, Y.M. (2015). Generalized Least Squares and Weighted Least Squares Estimation Methods for Distributional Parameters. REVSTAT-Statistical Journal, 13, 263-282.

Kantar, Y.M. and Yildirim, V. (2015). Robust Estimation for Parameters of the Extended Burr Type iii Distribution. Communications in Statistics-Simulation and Computation, 44, 1901-1930.

Kuo-Chao, L., W. Keng-Tung, C., Chien-Song, et al. (2009). A New Study on Combustion Behavior of Pine Sawdust Characterized by the Weibull Distribution. Chinese Journal of Chemical Engineering, 17, 860-868.

Lavanya, V., Rao, G.S. and Bidikar, B. (2016). Fast Fading Mobile Channel Modeling for Wireless Communication. Procedia Computer Science, 85, 777-781.

Lu, H.L. Chen, C.H. and Wu, J.W. (2004). A Note on Weighted Least-squares Estimation of the Shape Parameter of the Weibull Distribution. Quality and Reliability Engineering International, 20, 579-586.

Mohan, C.R., Rao, A.V. and Anjaneyulu, G.V.S.R. (2013). Comparison of Least Square Estimators with Rank Regression Estimators of Weibull Distribution-a Simulation Study. Journal of Statistics, 20.

Muraleedharan, G., Rao, A., Kurup, P., Nair, N.U. and Sinha, M. (2007). Modified Weibull Distribution for Maximum and Significant Wave Height Simulation and Prediction. Coastal Engineering, 54, 630-638.

Nadarajah, S. and Kotz, S. (2006). The Modified Weibull Distribution for Asset Returns. Quantitative Finance, 6, 449-449. 
Noga, K.M. and Palczynska, B. (2007). Overview of Fading Channel Modeling. International Journal of Electronics and Telecommunications, 56, 339-344.

Norman, L., Kotz, S. and Balakrishnan, N. (1994). Continuous Univariate Distributions.

Pascual, P. (2006). Morchella Esculenta (Morel) Rehydration Process Modeling. Journal of food Engineering, 72, 346-353.

Raghunathan, K., Subramaniam, V. and Srinivasamoorthy, V. (2002). Studies on the Tensile Characteristics of Ring and Rotor Yarns Using Modified Weibull Distribution.

Seki, T. and Yokoyama, S. (1996). Robust Parameter-estimation Using the Bootstrap Method for the 2-parameter Weibull Distribution. IEEE Transactions on Reliability, 45, 34-41.

Stankova, T.V. and Zlatanov, T.M. (2010). Modeling Diameter Distribution of Austrian Black Pine (Pinus Nigra Arn.) Plantations: a Comparison of the Weibull Frequency Distribution Function and Percentile-based Projection Methods. European Journal of Forest Research, 129, 1169-1179.

Surendran, P., Lee, J.H., Ko, S.J. et al. (2014). Parameter Optimization of uwb srr System Performance in Weibull Clutter Environment. Organization, 7.

Team, R.C. (2014). R: A Language and Environment for Statistical Computing. R Foundation for Statistical Computing, Vienna, Austria.

Teimouri, M., Hoseini, S.M. and Nadarajah, S. (2013). Comparison of Estimation Methods for the Weibull Distribution. Statistics, 47, 93-109.

Tiryakioglu, M. and Hudak, D. (2007). On Estimating Weibull Modulus by the Linear Regression Method. Journal of Materials Science, 42, 10173-10179.

Van Zyl, J.M. and Schall, R. (2012). Parameter Estimation Through Weighted Least-squares Rank Regression with Specific Reference to the Weibull and Gumbel Distributions. Communications in Statistics-Simulation and Computation, 41, 1654-1666.

Wang, F. and Keats, J.B. (1995). Improved Percentile Estimation for the Two-parameter Weibull Distribution. Microelectronics Reliability, 35, 883-892.

Wayne, N. (1982). Applied Life Data Analysis.

Wood, M.A., Gunderson, B.A., Zhou, X., Padmanabhan, V. and Ellenbogen, K.A. (2005). Temporal Patterns of Ventricular Tachyarrhythmia Recurrences Follow a Weibull Distribution. Journal of Cardiovascular Electrophysiology, 16, 181-185.

Zhang, L., Xie, M. and Tang, L. (2008). On Weighted Least Squares Estimation for the Parameters of Weibull Distribution. In Recent Advances in Reliability and Quality in Design, 57-84, Springer. 
Zhang, L., Xie, M. and Tang, L.C. (2007). A Study of Two Estimation Approaches for Parameters of Weibull Distribution Based on wpp. Reliability Engineering 83 System Safety, 92, 360-368.

\section{Sahar Sadani}

Department of Statistics,

Faculty of Sciences

Golestan University,

Gorgan, Iran.

email:s.sadani@gu.ac.ir

\section{Mahdi Teimouri}

Department of Mathematics and Statistics, Faculty of Sciences and Engineering

Gonbad Kavous University,

Gonbad Kavous, Iran.

email: teimouri@aut.ac.ir

\section{Kamel abdollahnezhad}

Department of Statistics,

Faculty of Sciences

Golestan University,

Gorgan, Iran.

email:k.abdollahnezhad@gu.ac.ir

\section{Vahid Ranjbar}

Department of Statistics,

Faculty of Sciences

Golestan University,

Gorgan, Iran.

email:v.ranjbar@gu.ac.ir 